\title{
Diagnosis and Management of Alzheimer Disease
}

\author{
Mel P. Daly, MD
}

Background: Alzheimer disease afllicts millions of older Americans, witl an estimated cost to society approaching $\$ 100$ million annually. Family physicians will care for an increasing number of patients with Alzheimer disease as well as their caregivers and families.

Methods: A comprehensive and systematic review of the literature published between 1985 and 1998 about diagnosing and treating Alzheimer disease was conducted, using "dementia," "Alzheimer's disease," and "treatment" as search strategy key words. Data and information that reported significant conclusions were critically reviewed. Potentially important new data about new agents that might be of benefit when caring for patients with Alzheimer disease are discussed.

Results and Conclusions: The primary goals when treating Alzheimer disease patients are enhancing autonomy and functional abilities and maintaining quality of life for patients and caregivers. In addition to diagnostic and pharmacologic treatment, primary care physicians will be called upon to provide nonpharmacologic support to assist with behavioral, social, and living environment problems faced by these patients and their families. The most common pharmacologic treatment is cholinesterase inhibition. Two cholinesterase inhibitors, tacrine and donepezil, are effective in treating cognitive and global function. Newer cholinesterase inhibitors should soon be available that might offer safety advantages as well as efficacy in treating behavioral and psychiatric symptoms related to Alzheimer disease. Other agents, including vitamin E, nonsteroidal anti-inflammatory drugs, estrogen, and Ginkgo biloba, are under investigation. Nonpharmacologic measures are important components in the management of Alzheimer disease. Support groups can help to diminish behavioral problems, maintain the patient's independence, and provide relief for caregivers and families. (J Am Board Fam Pract 1999;12:375-85.)

Dementia, the most common neurologic disorder affecting the elderly, is a syndrome characterized by loss of cognitive functioning sufficient to interfere with performing normal activities (eg, memory, calculation, abstract reasoning, language skills, and so on.). Changes in memory and attention occur with normal aging, and the degree to which these changes occur is variable. Older persons experience subtle age-associated changes in the rate at which new information is stored and in their ability to adjust rapidly to changing demands and to respond to new cognitive challenges. These changes, however, do not meet the criteria for dementia. ${ }^{1}$ Benign senescent forgetfulness that can occur in normal older persons is usually not associated with other cognitive effects and does not

Submitted, revised, 2 March 1999.

From the Departments of Family Medicine, Internal Medicine and Epidemiology \& Preventive Medicine, University of Maryland at Baltimore. Address reprint requests to Mel P. Daly, MD, Greater Baltimore Medical Center, Suite 5417, 6701 North Charles St, Baltimore, MD 21204.

This article was supported in part by a grant from Bayer Corporation. affect daily functioning. It might be difficult to distinguish normal age-related changes in cognitive functioning from the earliest manifestations of dementia.

Alzheimer disease, the most common cause of dementia, is a progressive neurologic disorder characterized by changes in behavior, personality, judgment, and the ability to perform usual activities. The classic triad for patients with Alzheimer disease is memory impairment, visuospatial defects, and language changes. These changes occur in the absence of confusion (unlike delirium), mental retardation, or other neurologic disorders and represent a considerable decline from a patient's usual level of functioning. ${ }^{2}$ About $10 \%$ (3.5 million) of persons older than 65 years are affected by Alzheimer disease. The prevalence of Alzheimer disease increases with each decade after 65 years, so that up to $40 \%$ of persons older than 85 years are affected. More than $65 \%$ of persons in nursing homes have cognitive impairment, and the most common cause is Alzheimer disease. A dramatic increase in the occurrence of Alzheimer disease is expected in the 
next century, when it is estimated that there will be 14 million persons with Alzheimer disease by the year $2040 .^{3}$

It is likely that most cases of dementia are initially seen in primary care settings. Despite its widespread occurrence, dementia often goes unrecognized or is misdiagnosed in its early stages. ${ }^{4}$ Alzheimer disease places a substantial burden on the health care system, which reports annualized costs of $\$ 100$ billion., ${ }^{5,6}$ Age and family history are major risk factors for $\mathrm{Alzheimer}$ disease. ${ }^{7}$ Fifty percent of persons who have relatives with Alzheimer disease will develop the disease by the time they are 90 years old. Other risk factors include previous head injury, female sex, lower educational level, and other genetic risks. ${ }^{8}$

\section{Methods}

This article set out to review existing information about diagnosing and treating Alzheimer disease. An extensive and systematic review of the literature was conducted by means of a MEDLINE search using "dementia," "Alzheimer's disease," and "treatment" as search strategy key words. The search covered the period between 1985 and 1998. Existing guidelines and expert opinions about diagnosing and treating Alzheimer disease were reviewed. Specific search strategies for the literature on treatment included "dementia" in the title field, together with "cholinesterase inhibitors" (donepezil and metrifonate) and "outcome" (daily life activity or other outcome of treatment).

Articles were reviewed for relevance, and priority was given to articles with the strongest study design that showed the most significant conclusions relative to diagnosing and treating Alzheimer disease. In the absence of direct evidence based on well-designed clinical trials in elderly persons, the opinions of authoritative bodies (eg, consensus conference statements, Agency for Health Care Policy Research policy practice guidelines) were included. New research data about treatment interventions for patients with Alzheimer disease were included to reflect emerging concepts about potentially beneficial treatments for patients with Alzheimer disease.

\section{Natural History}

Alzheimer disease most often occurs after the age of 60 years. Patients typically have a slowly pro- gressive decline of cognition. The course is vari- $\frac{c}{3}$ able, however, and most patients have a median life w expectancy of 10 to 15 years from diagnosis. ${ }^{9} \mathrm{Alz}-\frac{\mathrm{Q}}{0}$ heimer disease often results in despair for family members and caregivers, who must adjust to a $\Xi$ steady decline in function and behavior by the af- $\frac{0}{\mathbb{N}}$ flicted person. ${ }^{10} \mathrm{~A}$ decision to place a person with $\stackrel{?}{?}$ Alzheimer disease in a nursing home occurs most $\underset{\vec{\sigma}}{\vec{S}}$ often when incontinence or disruptive behaviors $\bar{C}$ (wandering, combativeness, agitation), become un- $\frac{}{0}$ manageable for the caregiver.

\section{Pathologenesis and Genetics}

The characteristic pathologic findings on postmortem examination of brains of patients with Alzheimer disease are neurofibrillary tangles, amyloid plaques, and neuritic plaques. A generalized loss of cortical cells is also characteristic. Neurofibrillary $\vec{N}$ tangle counts are associated with the severity of $\dot{\omega}$ dementia; however, neurofibrillary tangles can be of found in other degenerative disorders of the brain. $\stackrel{?}{?}$ Neurofibrillary tangles are made up of a protein $\mathcal{D}$ known as tau that becomes altered with Alzheimer disease. The distribution of neurofibrillary tangles $\underset{\nabla}{\stackrel{D}{7}}$ in Alzheimer disease is specific to the association cortex and spares the motor and sensory cortex. This finding correlates with clinical symptoms in patients with Alzheimer disease, whose executive functioning becomes impaired, yet motor and sensory symptoms occur later in the disease. $\beta$-Amyloid is normally a soluble substance, but in Alzheimer disease it becomes insoluble, forming extracellular deposits and neuritic plaques. $\beta$-Amyloid and neuritic plaques are specific to Alzheimer disease, yet they are only moderately correlated with severity of symptoms. Postulated pathophysiologic associations include free-radical release or disruptions of potassium or calcium channels.

Genetic mutations and chromosome abnormalities have been shown to be associated with an increased risk of Alzheimer disease. Persons with the apolipoprotein $\mathrm{E}$ type 4 (apoE-4) allele located on chromosome 19 are at increased risk for developing Alzheimer disease. The specificity for $\underset{\mathscr{N}}{\mathscr{W}}$ apoE-4 genotyping is low, so definitive genetic counseling is not possible. Thus screening for $\mathrm{Alz}-\frac{\bar{\Phi}}{\varnothing}$ heimer disease for apoE-4 genotyping is not recommended for the general population. Determining apoE-4 status is indicated only for patients with very unusual cases or in experimental or study situations. 
It is thought that a gene on chromosome 21 encodes for the amyloid precursor protein, a major component of amyloid deposits in Alzheimer disease and Down syndrome. Other research suggests that chromosomes 1, 14, and 21 might be linked to early-onset Alzheimer disease, whereas a gene on chromosome 19 might be associated with late-onset familial Alzheimer disease (LOFAD). ${ }^{11}$ This gene has three alleles (apoE-2, apoE-3, and apoE4). The presence of apoE- 4 occurs in $40 \%$ of patients with LOFAD and might be a marker for early detection. This allele, however, is also found in many elderly patients without Alzheimer disease and is not found in many patients with Alzheimer disease; thus, genetic testing for apoE is currently not recommended for routine screening. ${ }^{12}$ Some other forms of late-onset Alzheimer disease have been linked to chromosome $12 .^{13}$

\section{Clinical Findings}

Patients with Alzheimer disease have symptoms of cognitive impairment and an impaired ability to perform basic and instrumental activities of daily living. Early forgetfulness and gradual difficulties with executive functioning (ie, balancing checkbooks, complex decision making) occur, while social functioning is usually preserved. The ability to accomplish familiar tasks (driving, planning meals, using a telephone, self-care activities) is usually preserved in the early stages of Alzheimer disease. ${ }^{14}$ An inability to perform these activities might be the first sign for family members that something is awry. Behavioral changes, such as asking the same question repeatedly, inability to take messages, getting lost and wandering, and having difficulty doing usual tasks, are suggestive of a dementing illness. Visuospatial disturbances manifest as a propensity to get lost and copy instructions. Patients with Alzheimer disease usually have little insight into their impairments. In later stages patients might be unable to recall familiar objects or newly learned information. As the disease advances, patients might have behavioral symptoms, such as irritability, apathy, wandering, psychosis, disorientation, and agitation. Eventually, patients can become completely dependent.

\section{Diagnosis and Workup}

If patients or family members are concerned about cognitive decline, primary care physicians should
Table 1. Dementia Assessment.

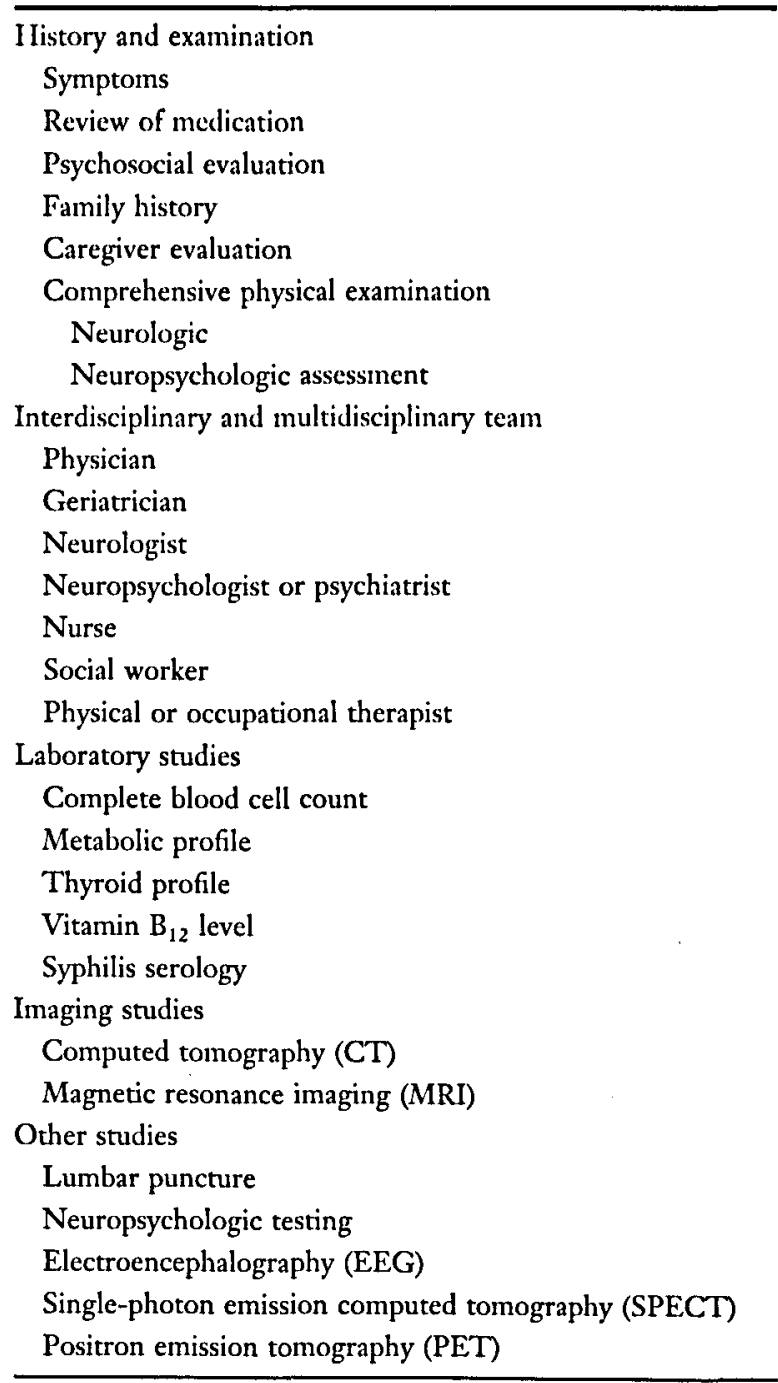

Adapted from Veterans Health Administration, ${ }^{18}$ Table 7: Components of Dementia Assessment Process.

consider the diagnosis of dementia. The most important diagnostic information is usually obtained by interviewing patients and caregivers and conducting a comprehensive clinical assessment (Table 1). The interview should address recent onset of symptoms, progression of symptoms, duration of symptoms, comorbid medical illness, medications ${ }^{15}$ (antidepressants, sedatives, anticonvulsants, antiParkinsonian drugs, antihypertensive agents, antihistamines, narcotics) and alcohol use, family history of Alzheimer disease, psychosocial history, social support networks, education, literacy, and living arrangements. The medical history should specifically include questions about known neurologic and psychiatric disorders, substance abuse, and exposure to environmental toxins. Changes in 
cognition should be compared with previous performances, and multiple dimensions of cognitive abilities (memory, abstract reasoning, spatial ability, handling complex tasks, language, and behavior) should be assessed. It is usually useful to ask close family members about their observations. A multidimensional assessment should always consider the possibility that family members or friends might exaggerate, deny, or minimize symptoms for different reasons.

A comprehensive physical examination focusing on a detailed neurologic and mental status examination is always indicated. Focal neurologic signs can suggest cerebrovascular disease. Dementias associated with physical illness, such as advanced cardiac or pulmonary disease, can occur as a result of reduced cerebral oxygen perfusion. These patients might show chronic cognitive impairments. Standardized mental status assessment instruments, such as the Mini-Mental State examination, 16 should be administered. This examination should include assessments of memory, attention, expressive and receptive language, orientation, and visual constructive ability. Although this scale is specific for diagnosing cognitive impairment, it might not be sufficiently sensitive compared with comprehensive neuropsychologic evaluations, especially among patients who are well educated. Serial administration of these screening tests allows for comparison over time and is likely to increase test sensitivity. Neuropsychologic testing is most useful early in the course of the disease or in differentiating Alzheimer disease from depression, delirium, or vascular dementias.

As soon as dementia is diagnosed, the next step is to determine the cause of cognitive impairment. In nearly all cases, the diagnosis can be made on the basis of a medical and psychiatric evaluation. In some cases the diagnosis is obvious (posttraumatic dementia, postanoxic dementia, or dementia resulting from a massive cerebrovascular accident), and no additional workup is required. Dementia associated with Huntington disease and Parkinson disease has characteristic associated symptoms and requires no further diagnostic evaluation. Among patients with dementia of uncertain cause or when dementia is progressive or manifests with atypical symptoms (aphasia), further evaluation is always indicated. Although there is no evidence to support the routine use of screening tests for Alzheimer disease, because no test has high enough sensitivity to detect early Alzheimer disease, testing is indi- $\frac{c}{3}$ cated once dementia is suspected. Some disorders that result in dementia are potentially reversible. $\frac{0}{2}$ Even though truly reversible dementia syndromes are relatively rare, many coexistent disorders that $\Xi$ might exacerbate symptoms of dementia or cause delirium can become apparent by laboratory or $\stackrel{?}{?}$ further diagnostic testing. Among the most frequent causes of reversible dementia with symptoms similar to those of Alzheimer disease are depression, alcohol abuse, and drug toxicity. If dementia is suspected to be caused by medications, alcohol, or is drugs, further evaluation is indicated should symp- $\vec{\circ}$ toms persist after discontinuing the potentially causative agent. Most of these patients will require longitudinal follow-up care.

The laboratory evaluation should include a complete blood cell count, blood chemistry analysis $\stackrel{\sim}{\circ}$ (including calcium), a serologic test for syphilis, thyroid function tests (thyroid-stimulating hormone and free thyroid index), liver function tests, and assays of vitamin $B_{12}$ and red blood cell folate levels. In many cases, a noncontrast computed tomographic (CT) head scan is appropriate, particularly when there are focal neurologic deficits. These basic laboratory assessments will detect anemia, diabetes, renal and liver disease, thyroid disease, vitamin deficiency, and syphilitic infections that could be causing or contributing to the patient's dementia. Follow-up assessments at periodic intervals could be the most useful diagnostic procedure for differentiating Alzheimer disease from normal aging.

Lumbar puncture is not a routine diagnostic test for persons with dementia but should be done if metastatic cancer, meningitis, tertiary syphilis, hydrocephalus, encephalitis, demyelinating disease, immunosuppression, or inflammatory disease is suspected. Future cerebrospinal fluid studies might be useful in diagnosing Alzheimer disease (decreased $\beta$-amyloid, increased tau concentrations, or presence of neuronal thread proteins).

Neuropsychologic testing, in general, should be reserved for patients who have abnormal mental status test results and normal physical functioning, or when serious concerns about dementia are raised by family members, yet results of screening tests are normal. This evaluation consists of well-standardized tests of cognitive functioning, including reasoning and problem solving, language, visuospatial skills, attention, memory, naming, and intelli- 
gence. This level of testing can provide accurate qualitative and quantitative assessments of cognition and can be used to predict rates of cognitive declines. ${ }^{17}$ Neuropsychologic testing should be interpreted in the context of other historical and clinical information. Follow-up testing at intervals of 6 to 9 months is most useful for patients with symptoms of dementia but in whom the diagnosis is uncertain. Brain scanning is not necessary if the cause for dementia can be diagnosed with reasonable certainty based on the history, physical examination, and laboratory studies.

For patients with recent-onset dementia, focal neurologic signs, or atypical symptoms, or among those for whom the diagnosis is uncertain, neuroimaging studies can help in the diagnostic evaluation. Pathologic findings shown by CT scanning or magnetic resonance imaging (MRI) include brain tumors, abscesses, strokes, hematomas, arteriovenous malformations, and hydrocephalus. Findings on CT or MRI scans can suggest frontotemporal dementias, Huntington disease, progressive supranuclear palsy, and ataxia syndromes. Unusual basal ganglia disorders that might produce CT or MRI changes include Wilson disease and basal ganglion disease. The role of diagnostic imaging studies, such as single-photon emission computed tomography (SPECT), positron emission tomography (PET), and weighted MRI, has not been fully determined. These studies are most useful in diagnosing vascular dementias and dementias with atypical symptoms; however, the usefulness of PET and SPECT scanning in determining the cause of dementia in heterogeneous populations has not been established.

\section{Differential Diagnosis}

The diagnosis of Alzheimer disease can usually be based on symptoms and diagnostic tests. Other causes that should be considered in the differential diagnosis include multi-infarct dementia (up to $15 \%$ of all patients), Parkinson disease, Pick disease, Huntington chorea, normal-pressure hydrocephalus, metabolic disorders (vitamin $\mathrm{B}_{12}$ deficiency, alcoholism, hypothyroidism, and drug intoxication), infectious causes (neurosyphilis, human immunodeficiency virus, encephalitis, meningitis), dementia associated with diffuse Lewy bodies, and depression.

The diagnosis of dementia requires integrating findings determined by history, physical examina- tion, neuropsychologic testing, laboratory studies, and neuroimaging studies. ${ }^{18}$ Patients with Alzheimer disease usually have normal findings on neurologic examinations until very advanced stages of the disease. Focal neurologic signs indicate the presence of focal brain lesions that might suggest multi-infarct dementia (including such rare disorders as multiple sclerosis or brain abscesses). Subcortical disorders (Parkinson disease, progressive supranuclear palsy, Lewy body disease, strionigral degeneration, and neuroleptic medications) typically cause memory deficits, intact language, and impaired motor functioning and executive functioning. Subcortical vascular dementia (Binswanger disease) is caused by arteriosclerosis of arterioles of the midbrain, frequently occurring among patients with hypertension, diabetes mellitus, and hypercholesterolemia. In these patients, CT or MRI findings are usually nonspecific.

Frontal lobe dementias (Pick disease, frontotemporal lobe atrophy with Pick bodies, or motor neuron disease) are associated with early onset of behavioral changes, marked changes in judgment, and mild defects in memory and visuospatial orientation. Disinhibition, compulsiveness, impatience, sexual inappropriateness, and lability of affect are prominent symptoms. Neuroimaging studies can help in accurately diagnosing these dementias. Diffuse Lewy body dementia manifests with symptoms similar to those of Alzheimer disease but with prominent symptoms of hallucinations, mild parkinsonian symptoms, mental status changes, and sensitivity to neuroleptic drugs. It is uncertain whether this clinical entity is new, because many patients with Lewy body dementia have coexisting Alzheimer disease. ${ }^{19}$

The finding on physical examination of myoclonic jerks can suggest Creutzfeldt-Jacob disease, a rare, rapidly progressive dementing illness. Myoclonus can also be found with dementia associated with anemia or liver failure. The triad of gait apraxia, dementia, and urinary incontinence suggests normal-pressure hydrocephalus, although many patients have vascular dementia in association with Alzheimer disease.

Patients with Alzheimer disease can have symptoms of depression. These patients usually have a sad or blunted affect and often express neurovegetative symptoms and feelings of worthlessness and hopelessness. Patients with depression are usually more impaired in abilities to make and carry out 
plans, concentration, and attention, whereas patients with Alzheimer disease usually have marked visuospatial impairments. These patients might minimize their cognitive deficits, unlike patients with depression, who typically are unwilling to answer cognitive test questions and usually have cognitive complaints that exceed measured deficits. $\mathrm{Pa}$ tients with depression can also have cognitive deficits associated with pain and physical complaints. There might be a history of previous depression or a family history of depression; cognitive symptoms are usually of recent onset. Depression and Alzheimer disease often overlap, and the diagnosis might be confirmed by a therapeutic trial of antidepressant medications. For patients who have Parkinson's disease, tremor and rigidity precede cognitive symptoms by years, unlike diffuse Lewy body dementia, which is characterized by hallucinations and alterations in alertness and attention occurring concurrently with parkinsonian signs. Patients with alcohol-related dementia typically confabulate and have preserved social skills.

\section{Treatment}

The goal of caring for patients with Alzheimer disease is to enhance function, maintain quality of life, and preserve autonomy. This goal can be achieved through high-quality primary care, including regular (4- to 6-month) reevaluations, education of and frequent contact with family members and caregivers, and careful attention to general health. High-quality health care makes it possible to delay and prevent the onset of illness and later to treat behavioral symptoms, cognitive deficits, progression of illness, and medical co-morbidities. The optimal approach to caring for patients with Alzheimer disease addresses medical, psychologic, and social needs of patients and caregivers. Because symptoms are progressive and the needs of patients and caregivers change with time, care must be longitudinal. Nonpharmacologic interventions, such as social support (Alzheimer's Association support groups, adult day care, respite care, and long-term care), family education, and environmental enhancement, are important at all stages. For instance, changing the home and environment can enhance safety and improve quality of life. Patients and family members need advice about a patient's ability to drive and make complex decisions about financial matters, and they need to consider ad- vance directives. Information can be obtained by $\stackrel{c}{\longrightarrow}$ contacting regional or national support organizations (Alzheimer's Association, Alzheimer's Disease Education and Referral Center, Administration on $\frac{\widehat{a}}{\pi}$ Aging, Children of Aging Parents).

As the condition progresses, increasing levels of $\frac{0}{0}$ supervision and support will prevent injury and $\stackrel{\Re}{?}$ help with activities of daily living. Supervision com- $\overrightarrow{\vec{c}}$ pensates for cognitive deficits at home, or patients $\frac{\overrightarrow{0}}{0}$ might require more restrictive living arrangements $\frac{\bar{\sigma}}{\bar{\omega}}$ (assisted living, adult day care, special Alzheimer $\stackrel{\bar{\sigma}}{\vec{\sigma}}$ disease units). Caregivers benefit by regular avail- $\frac{\varrho}{\infty}$ able and affordable respite care for care recipients. $\frac{\text { o }}{\partial}$ In-home help directed toward the patient allows caregivers more independence and provides time $\vec{N}_{N}$ for caregivers to spend high-quality time with pa- $\frac{\vec{\partial}}{\overline{0}}$ tients. Families caring for patients with Alzheimer $\stackrel{\vec{?}}{\overrightarrow{7}}$ disease often incur a substantial financial burden, so $\vec{N}$ patients and caregivers need ongoing advice about $\stackrel{\omega}{\omega}$ business and financial decisions. Family members ov are frequently called upon to provide unpaid care $\stackrel{5}{-}$ that can create considerable physical and psycho- $\vec{D}$ logical stress on the patient-caregiver dyad.

Ultimately, many patients will spend some time in nursing homes, usually because they need professional care for incontinence or behavioral out $-\vec{\varepsilon}$ bursts or because the caregiver needs relief from the physical or psychologic strain of caring for the patient.

Psychiatric symptoms associated with Alzheimer disease are common and distressing for patients and caregivers. Symptoms are usually depressive, anxious, psychotic, agitated, apathetic, or vegetative in nature. Interventions, such as environmental manipulation, structured activities, attention to sleep, and interventions to keep patients stimulated, can be effective. Many approaches have been attempted to manage behavioral problems associated with Alzheimer disease. Useful strategies include changing patient experiences frequently (redirection, al- $\stackrel{\bigcirc}{3}$ ternative activities), modifying the environment (use of night lights, clocks, calendars, family pictures, reminiscence), reducing choices (clothes, closets, menus), modifying eating arrangements $\underset{\omega}{\mathcal{N}}$ (eating with one or two companions instead of congregate eating), modifying external entrainers $\stackrel{0}{\stackrel{D}{0}}$ (noise, people talking outside the patient's room, sirens, and alarms), and providing caregiver advice (calmness, validation, distraction, and providing continuous supervision). Interventions such as re- $\mathbb{\otimes}$ ality orientation and memory retraining are of con- 
Table 2. Drugs Used to Treat Behavioral Symptoms in Patients with Dementia.

\begin{tabular}{|c|c|c|}
\hline Symptom & Drug & Dose \\
\hline Psychosis & $\begin{array}{l}\text { Ialoperidol } \\
\text { Thioridazine } \\
\text { Risperidone } \\
\text { (Others: olanzapine, divalproex) }\end{array}$ & $\begin{array}{l}0.5-2 \mathrm{mg} \text {, twice a day } \\
50-100 \mathrm{mg} \text {, twice a day } \\
0.5-3 \mathrm{mg} \text {, twice a day }\end{array}$ \\
\hline Agitation & $\begin{array}{l}\text { Haloperidol } \\
\text { Lorazepam } \\
\text { Trazodone } \\
\text { Carbamazepine }\end{array}$ & $\begin{array}{l}0.5-3 \mathrm{mg} \text {, twice a day } \\
0.5-3 \mathrm{mg} \text {, twice a day } \\
50-150 \mathrm{mg} \text {, twice a day } \\
200-400 \mathrm{mg} \text {, twice a day }\end{array}$ \\
\hline Anxiety & $\begin{array}{l}\text { Lorazepam } \\
\text { Buspirone } \\
\text { Propranalol } \\
\text { (Others: trazodone, SSRIs) }\end{array}$ & $\begin{array}{l}0.5-3 \mathrm{mg} \text {, twice a day } \\
5-15 \mathrm{mg} \text {, every day } \\
20-60 \mathrm{mg} \text {, three times a day }\end{array}$ \\
\hline Insomnia & $\begin{array}{l}\text { Lorazepam } \\
\text { Tamazepam } \\
\text { Zolpidem } \\
\text { Trazodone }\end{array}$ & $\begin{array}{l}0.5-2 \mathrm{mg} \text {, at bedtime } \\
15-30 \mathrm{mg} \text {, at bedtime } \\
5-10 \mathrm{mg} \text {, at bedtime } \\
50-100 \mathrm{mg} \text {, at bedtime }\end{array}$ \\
\hline Depression & $\begin{array}{l}\text { Nortriptyline } \\
\text { SSRIs (sertraline, paroxetine, etc) } \\
\text { (Others: electroconvulsive shock in selected } \\
\text { patients) }\end{array}$ & 25-150 mg, every day \\
\hline Sundowning & $\begin{array}{l}\text { Trazodone } \\
\text { Haloperidol } \\
\text { (Others: Risperidone, Olanzapine) }\end{array}$ & $\begin{array}{l}50-150 \mathrm{mg}, \text { at bedtime } \\
0.5-3 \mathrm{mg} \text {, at bedtime }\end{array}$ \\
\hline $\begin{array}{l}\text { Aggression (not due to psychosis, anxiety, } \\
\text { depression, or insomnia) }\end{array}$ & $\begin{array}{l}\text { Trazodone } \\
\text { Buspirone } \\
\text { Risperidone } \\
\text { (Others: olanzapine, carbamazepine, SSRIs) }\end{array}$ & $\begin{array}{l}50-150 \mathrm{mg} \text {, three times a day } \\
0.5-10 \mathrm{mg} \text {, three times a day } \\
0.5-0.3 \mathrm{mg} \text {, twice a day }\end{array}$ \\
\hline
\end{tabular}

SSRIs-selective serotonin reuptake inhibitors.

troversial benefit. ${ }^{20}$ Family caregivers can experience great benefits from becoming involved in supportive and educational activities. Carefully monitored physical restraints should be applied only in situations where patients are severely agitated despite all supportive interventions. Family physicians should counsel patients with Alzheimer disease and their family members about advance directives and decisions concerning terminal care (quality of life, institutionalization, feeding, and palliative care). ${ }^{21}$

Pharmacologic interventions can be appropriate for recalcitrant behaviors. (Table 2). Pharmacologic treatment of behavioral outbursts can reduce distress, decrease caregiver burden, and delay institutionalization. Drugs should be prescribed judiciously for patients with Alzheimer disease. The physician should start therapy with low doses and titrate gradually while observing for side effects and deleterious drug interactions. Antipsychotic medications are most effective for psychotic symptoms, and agents should be chosen based on a profile of potential side effects. ${ }^{22}$

High-potency drugs, such as haloperidol (I Ialdol) often cause tremor rigidity, akathisia, and rarely, tardive dyskinesia. Lower potency agents, such as thioridazine (Mellaril) can cause sedation, postural hypotension, and anticholinergic effects (dry mouth, blurred vision, urine retention). Newer drugs, such as clozapine, ${ }^{23}$ risperidone, ${ }^{24}$ and olanzapine, are potentially as effective and appear to have fewer extrapyramidal side effects. Benzodiazepines are effective for treating anxiety or agitation. Short-acting agents (oxazepam, lorazepam) are less likely to cause side effects (confusion, sedation, amnesia, and disinhibition) that are associated with longer acting drugs. For patients with behavioral symptoms not responding to these interventions, 
anticonvulsants, trazodone (for sleep and sedation), $\beta$-blockers, carbamazepine, buspirone, sodium valproate, or selective serotonin reuptake inhibitors can be effective. ${ }^{25}$

\section{Cholinesterase Inbibitors}

Cognitive dysfunction in patients with Alzheimer disease is directly associated with loss of cholinergic neurons. Acetylcholine is an important neurotransmitter that links the forebrain to the hippocampus, cortex, and amygdala (essential for memory and information processing). Acetylcholine concentrations decline in normal persons but decrease by up to $90 \%$ with Alzheimer disease. Acetylcholine is hydrolyzed by the enzyme acetylcholinesterase (AChE). Recently it has been shown that enhancing cholinergic functioning might attenuate the rate of cognitive loss among patients with Alzheimer disease.

Two agents are available for treating cognitive impairment associated with Alzheimer disease. Tacrine was the first AChE inhibitor available to treat mild-to-moderate Alzheimer disease. Tacrine is a centrally active aminoacridine with reversible $\mathrm{AChE}$ inhibitory activity and has a duration of activity of less than 8 hours. In clinical trials of more than 2000 patients with mild-to-moderate Alzheimer disease, $20 \%$ to $30 \%$ of patients treated with tacrine showed improvements in cognition when compared with placebo. ${ }^{26}$ Tacrine must be administered four times a day because of its short half-life, and it requires titrating of dosing to usually effective doses $(160 \mathrm{mg} / \mathrm{d})$ for a minimum period of 18 weeks. Up to $30 \%$ of patients experienced serious adverse reactions, including cholinergic effects (gastrointestinal distress, insomnia) and elevations of liver enzymes, frequently requiring discontinuing the medication. ${ }^{27}$ Whereas tacrine appears to reduce deterioration in cognitive performance and increase global clinical performance in patients with Alzheimer disease, the clinical relevance and long-term effects remain controversial.

A second-generation $\mathrm{AChE}$ inhibitor, donepezil (Aricept) is now available for treating cognitive impairment related to Alzheimer disease. This agent has a longer duration of AChE inhibitory action and has more specificity for brain tissue than tacrine. ${ }^{28}$ In clinical trials donepezil enhanced cognitive function in most patients, as rated by the Alzheimer's Disease Assessment Scale Cognitive Subscale (ADAS-Cog), and did not cause hepato- toxicity. Patients who discontinued donepezil had a loss of treatment benefit. Donepezil is well tolerated, especially at the starting dose of $5 \mathrm{mg} / \mathrm{d}$. The recommended higher dose $(10 \mathrm{mg} / \mathrm{d})$ usually requires a 4- to 6-week titration period and can cause 3 cholinergic-mediated side effects, (nausea, diar- $\frac{7}{0}$

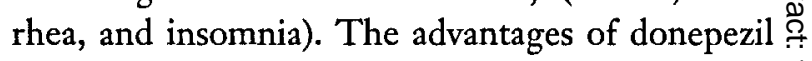
compared with tacrine are that it may be given once $\underset{\vec{S}}{\vec{S}}$ a day, it does not require as long an introductory $\overrightarrow{0}$ dosing period, and there is no need to monitor liver $\frac{\bar{\sigma}}{\bar{\omega}}$ function regularly. In clinical trials no drug inter- $\frac{\vec{\phi}}{\mathrm{D}}$ actions were observed with digoxin, warfarin, theophylline, and cimetidine. Donepezil is metabo- $\overrightarrow{0}$ lized by the cytochrome P-450 system, so caution should be used when medications metabolized by this system are prescribed. The most common ad- $-\widehat{\vec{\sigma}}$ verse effects are nausea, diarrhea, insomnia, vom- $\overrightarrow{3}$ iting, muscle cramps, fatigue, and anorexia. These $\vec{N}$ events were of mild intensity, transient, and rarely $\dot{\omega}$ required dose modification.

Clinical trials of other agents to improve cognitive functioning are ongoing, including, metrifon- $\bar{\Phi}$ ate, rivastigmine, physostigmine, and others. ${ }^{29}$ The $\frac{\mathbb{O}}{\mathbb{1}}$ most widely-studied agent has been metrifonate, 큼 which has no intrinsic anti-AChE activity; how- $\stackrel{\mathbb{Q}}{\longrightarrow}$ ever, it is transformed to an active compound $(2,2-\vec{\emptyset}$ dimethyl-dichlorvinyl phosphate [DDVP]), a potent inhibitor of AChE. When compared with other AChE inhibitors (tacrine, physostigmine), 产 metrifonate achieved higher levels of AChE inhi- ٌ bition with fewer side effects, had a longer duration $\stackrel{\bigcirc}{\rightarrow}$ of action, and maintained the steady state of ace- $\frac{\rho}{3}$ tylcholine in the brain. ${ }^{30}$ A prospective, 30 -week, multicenter, double-blind, randomized, placebocontrolled, dose-finding study of three once-daily doses of metrifonate in patients with probable $\mathrm{Alz}$ heimer disease showed noticeable improvements in ADAS-Cog scores and Clinician's Interview Based Impression of Change with Caregiver Input (CIBIC-plus) scores. The drug was well tolerated, $\stackrel{\bigcirc}{\sim}$ and the side-effect profile was favorable. Most side $\stackrel{\circ}{\circ}$ effects were gastrointestinal. These study findings suggest that metrifonate might be important in the $\tilde{\sim}$ treatment of Alzheimer disease. ${ }^{31}$ Metrifonate is $\stackrel{N}{\omega}$ well-tolerated with mild, transient side effects, and ${ }^{2}$ little to no accumulation of metrifonate or DDVP is found with chronic dosing. ${ }^{32}$

\section{Estrogens}

It has been hypothesized that reduced levels of $\stackrel{\vec{\Phi}}{\stackrel{\mathbb{Q}}{\circ}}$ estrogens after menopause are associated with an 
increased risk for Alzheimer disease, and that estrogen replacement therapy might lower the risk of developing Alzheimer disease. ${ }^{33}$ The protective effects of estrogen might be mediated through effects on blood flow, $\beta$-amyloid deposition, or neurotropic actions. Epidemiologic evidence supporting the benefits of estrogen therapy is so far inconclusive. Studies of estrogen therapy to prevent or improve cognitive functioning are mostly based on retrospective case-control findings that are confounded by inherent sampling differences, such as lifestyle and other differences between estrogen users and control subjects. It could be that estrogen does not prevent the onset of Alzheimer disease but delays its expression. Furthermore, there is little evidence to suggest that there is a sex difference in the prevalence or incidence of Alzheimer disease. Only through randomized, placebo-controlled intervention trials can the efficacy of estrogen be truly determined. Large samples (7000 to 8000 women in each arm) will be required to show a protective or therapeutic effect (to reduce the likelihood of a type II statistical error). ${ }^{34}$ Further studies are indicated to assess the safety of estrogen replacement therapy in postmenopausal women. ${ }^{35}$

\section{Nonsteroidal Anti-inflammatory Drugs}

Recent evidence suggests that nonsteroidal antiinflammatory drugs (NSAIDs) (aspirin, ibuprofen) can slow the onset or progression of Alzheimer disease, by reducing inflammation in the brain, which results in the deposition of amyloid proteins, characteristic of Alzheimer disease. ${ }^{36}$ The role of NSAIDs has been supported by retrospective studies showing that patients using long-term NSAIDs have a reduced incidence of Alzheimer disease compared with those not receiving NSAIDs. ${ }^{37}$ Studies are ongoing to evaluate the efficacy of NSAIDs in slowing the progression of Alzheimer disease. Concerns about the use of NSAIDs in the elderly include potential toxicities, particularly those that are gastrointestinal and renal.

\section{other Agents}

Other agents, such as vitamin $\mathrm{E}$ ( $\alpha$-tocopherol) ${ }^{38}$ and selective monoamine oxidase- $B$ inhibitors (selegiline), and neurotropic growth factors, which might potentially enhance acetylcholine levels in patients with Alzheimer disease (these agents would require intracerebroventricular administration), are under investigation. These agents might be useful in treating Alzheimer disease by modifying pathophysiologic effects or by attenuating rates of degeneration of cholinergic-producing cells. Vitamin $\mathrm{E}$ is associated with few or no side effects in doses of $2000 \mathrm{IU} /$ d. Ginkgo biloba extract has recently been reported to delay symptom progression in patients with Alzheimer disease, perhaps through its antioxidant effects. Patients taking ginkgo extract for 1 year in a randomized, double-blind study, showed improvement in cognition (ADAS-Cog scores) when compared with placebo. ${ }^{39}$ This study estimated that 7 patients need to be treated with 120 $\mathrm{mg}$ of gingko extract for 1 year for one of them to have an improved ADAS-Cog score of four points that they would not have had with placebo. Little is known about long-term efficacy or side effects.

\section{Conclusion}

Family physicians can diagnose, treat, and manage most cases of Alzheimer disease. Despite advances in the development of new treatments for Alzheimer disease, many patients and caregivers receive inadequate care. Family physicians should be alert for Alzheimer disease when patients or caregivers describe functional declines. Patients and family members might not seek assistance because they are reluctant to admit to deficits and tend to compensate for them. Family involvement is critical, and physician counseling, education, and support cannot be underestimated.

Questions remain about current and investigational therapies, and the appropriate modalities and settings for caring for patients with Alzheimer disease. Strategies for the family physicians to adopt might include regular visits, education and support for caregivers, anticipatory guidance, and strategies to address agitation (wandering and unsafe driving). Many patients and caregivers benefit from adult day care and respite care, especially in later stages of the disorder. Community support from neighbors and friends, home care agencies, mobile meal programs, homemakers, and volunteer visitors can be invaluable at all stages to delay the onset and slow the progression of Alzheimer disease symptoms.

Pharmacologic interventions are being investigated. Disease-modifying agents have been studied, and newer agents show considerable safety and potential cognitive-enhancing benefits. Anticholinesterase inhibitors can improve and reduce the rate of 
decline of cognitive functioning. The newer cholinesterase inhibitors have improved safety and dosing convenience. These agents have been effective in treating the cognitive aspects of Alzheimer disease; some have also been effective in treating behavioral and psychiatric symptoms of Alzheimer disease. Further studies of risk factors, prevention, early recognition, access to care, and treatment (pharmacologic, social, behavioral, and diseasemodifying strategies) will be of major public health importance in the new millennium.

\section{References}

1. Cullum CM, Rosenberg RN. Memory loss-when is it Alzheimer disease? JAMA 1998;279:1689-90.

2. Diagnostic and statistical manual of mental disorders: DSM-IV. 4th edition. Washington, DC: American Psychiatric Press, 1994.

3. Evans DA. Estimated prevalence of Alzheimer's disease in the United States. Milbank Q 1990;68:26789.

4. Ross GW, Abbott RD, Petrovitch H, et al. Frequency and characteristics of silent dementia among elderly Japanese-American men. The Honolulu-Asia Aging Study. JAMA 1997;277:800-5.

5. Progress report on Alzheimer's disease 1996. Bethesda, Md: National Institutes of Health, National Institute on Aging, 1996. NIH publication 96-4137.

6. Ernst RL, Hay JW. The US economic and social costs of Alzheimer's disease revisited. Am J Public Health 1994;84:1261-4.

7. Mohs RC, Breitner JC, Silverman JM, Davis KL. Alzheimer's disease. Morbid risk factor among firstdegree relatives approximates $50 \%$ by 90 years of age. Arch Gen Psychiatry 1987;44:405-8.

8. Ganguli M, Ratcliff G, Huff FJ, Belle S, et al. Effects of age, gender, and education on cognitive tests in a rural elderly community sample: norms from the Monongahela Valley Independent Elders Survey. Neuroepidemiology 1991;10:45-52.

9. Jost BC, Grossberg GT. The natural history of Alzheimer's disease: a brain bank study. J Am Geriatr Soc 1995;43:1248-55.

10. Schulz R, O’Brien AT, Bookwala J, Fleissner K. Psychiatric and physical morbidity effects of dementia caregiving: prevalence, correlates, and causes. Gerontologist 1995;35:771-91.

11. Corder EH, Saunders AM, Strittmatter WJ, et al. Gene dose of apolipoprotein E type 4 allele and the risk of Alzheimer's disease in late onset families. Science 1993;261:921-3.

12. Apolipoprotein $\mathrm{E}$ genotyping in Alzheimer's disease: National Institute on Aging/Alzheimer's Association Working Group. Lancet 1996;347:1091-5.

13. Stephenson J. Researchers find evidence of a new gene for late-onset Alzheimer disease. JAMA 1997; 277:775.

14. Recognition and initial assessment of Alzheimer's disease and related dementias. Clinical practice guideline 19. Rockville, Md: Department of Health and Human Services, Public Health Service, Agency for Health Care Policy and Research, 1996. AHCPR publication 97-0702.

15. Morrison RL, Katz IR. Drug-related cognitive im- $\stackrel{\overrightarrow{\vec{D}}}{\overrightarrow{0}}$ pairment: current progress and the current prob- 0 lems. Ann Rev Gerontol Geriatr 1989;9:232-79.

16. Folstein MF, Folstein SE, McHugh PR. Mini-men- $\frac{\vec{D}}{\mathrm{D}}$ tal state: a practical method for grading the cognitive state of patients for the clinician. J Psychiatr Res ${ }^{\text {s }}$ 1975;12:189-98.

17. Cullum CM, Filley CM, Kozora E. Episodic mem- $-\frac{\omega}{N}$ ory function in advanced aging and early Alzheimer's $\stackrel{N}{\stackrel{N}{\circ}}$ disease. J Int Neuropsychol Soc 1995;1:100-3.

18. Dementia identification assessment: guidelines for $\frac{\vec{N}}{N}$ primary care practitioners. Washington, DC, and $\tilde{N}$ Oak Brook, Ill: Veterans Health Administration; $\omega$ University HealthSystem Consortium, 1997:17. ज Adapted from Table 7 Components of Dementia Assessment Process.

19. McKeith LG, Galasko D, Kosaka K, et al. Consensus $\frac{\mathbb{D}}{\square}$ guidelines for the clinical and pathologic diagnosis of $\stackrel{\mathbb{D}}{3}$ dementia with Lewy bodies (DLB): report of the $\underset{\Phi}{\Phi}$ consortium on DLB international workshop. Neurology 1996;47:1113-24.

20. Baines S, Saxby P, Ehlert K. Reality orientation on reminiscence therapy: a controlled cross-over study of elderly confused people. Br J Psychiatry 1987;151: 222-31.

21. Beck CK. Psychosocial and behavioral interventions for Alzheimer's disease patients and their families. Am J Geriatr Psychiatry 1998;6(2 Suppl 1):S41-8.

22. Treatment of agitation in older persons with dementia. Postgrad Med 1998;Spec No:1-88.

23. Salzman C, Vacarro B, Lieff J, et al. Clozapine in older patients with psychosis and behavioral disturbances. Am J Geriatr Soc 1995;3:26-33.

24. Madhusoodanan S, Brenner R, Araujo L, Abaza A. 웅 Efficacy of risperidone treatment for psychoses as- $O$ sociated with schizophrenia, schizoaffective disorder, N bipolar disorder, or senile dementia in 11 geriatric patients: a case series. J Clin Psychiatry 1995;56: 을. 514-8.

25. Small GW, Rabins PV, Barry PP, Buckholtz NS, et $\stackrel{\mathcal{\omega}}{\omega}$ al. Diagnosis and treatment of Alzheimer disease and $\bar{Z}$ related disorders: Consensus statement of the Amer- 0 ican Association for Geriatric Psychiatry, the Alzheimer's Association, and the American Geriatrics So- $\stackrel{+}{+}$ ciety. JAMA 1997;278:1363-71.

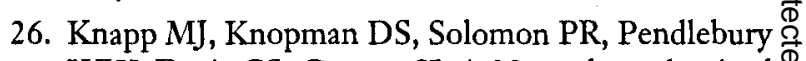
WW, Davis CS, Gracon SI. A 30-week randomized $\stackrel{\mathbb{}}{\square}$ controlled trial of high-dose tacrine in patients with 
Alzheimer's disease: The Tacrine Study Group. JAMA 1994;271:985-91.

27. Schneider LS. Clinical pharmacology of aminoacridines in Alzheimer's disease. Neurology 1993; 43(Suppl 14):S64-S79.

28. Rogers SL, Friedhoff LT. The efficacy and safety of donepezil in patients with Alzheimer's disease: results of a US multicenter, randomized, double-blind, placebo-controlled trial. The Donepezil Study Group. Dementia 1996;7:293-303.

29. Hallak M, Giacobini E. Physostigmine, tacrine and metrifonate: the effect of multiple doses on acetylcholine metabolism in rat brain. Neuropharmacology 1989;28:199-206.

30. Becker RE, Colliver J, Elble R, et al. Effects of metrifonate, a long-acting cholinesterase inhibitor in Alzheimer's disease: report of an open trial. Drug Dev Res 1990;19:425-34.

31. Cummings JL, Cyrus PA, Bieber F, Mas J, Orazem, J, Gulanski B. Metrifonate treatment of the cognitive deficits of Alzheimer's disease. Metrifonate Study Group. Neurology 1998;50:1214-21.

32. Pettigrew LC, Bieber F, Lettieri J, et al. Pharmacokinetics, pharmacodynamics, and safety of metrifonate in patients with Alzheimer's disease. J Clin Pharmacol 1998;38:236-45.

33. Kawas C, Resnick S, Morrison A, et al. A prospective study of estrogen replacement therapy and the risk of developing Alzheimer's disease: the Baltimore Longitudinal Study of $\Lambda$ ging. Neurology 1997;48:151721.

34. Kuller LII. Hormone replacement therapy and its potential relationship to dementia. J Am Geriatr Soc 1996;44:878-80.

35. Tang MX, Jacobs D, Stern Y, et al. Effect of oestrogen during menopause on risk and age at onset of Alzheimer's disease. Lancet 1996;348:429-32.

36. Stephenson J. More evidence links NSAID, estrogen use with reduced Nzheimer risk. JAMA 1996;275: 1389-90.

37. Tariot PN, Schneider L. Contemporary treatment approaches to Alzheimer's disease. Consult Pharm 1996;11:(Suppl E):16-24.

38. Sano M, Ernesto C, Thomas RG, et al. A controlled trial of selegiline, alpha-tocopherol, or both as treatment for Alzheimer's disease. The Alzheimer's Disease Cooperative Study. N Engl J Med 1997;336: 1216-22.

39. LeBars PL, Katz MM, Berman N, Itil TM, Freedman AM, Schatzberg AF. A placebo-controlled, double-blind, randomized trial of an extract of Gingko biloba for dementia. North American EGb Study Group. JAMA 1997;278:1327-32. 